\title{
WIMP Dark Matter as an active component
}

Fabio locco*

Marie-Curie fellow; Institut d'Astrophysique de Paris, UMR 7095-CNRS Paris, Université

Pierre et Marie Curie, 98bis bd Arago, F-75014, Paris, France

E-mail: ioccodiap.fr

In these proceedings I briefly review the main mechanisms by means of which Weakly Interacting Massive Particles, here assumed to constitute Dark Matter, can contribute beyond the pure gravitational interaction to the evolution of the Universe. In particular, I will comment on how they can modify the yields of Primordial Nucleosynthesis, alter the Reionization history and take part into the first stellar episode in the Universe.

International Workshop on Cosmic Structure and Evolution - Cosmology2009,

September 23-25, 2009

Bielefeld, Germany

${ }^{*}$ Speaker. 


\section{Introduction}

Observational evidence of diverse nature strongly hints toward the existence of a matter component of the Universe, so far undetected in the electromagnetic spectrum, but only via its contribution to gravitational signatures. Whereas there is quite general agreement about the existence of such a dark matter component, yet its nature is unknown. Primordial Nucleosynthesis (BBN) and Cosmic Microwave Background (CMB) based arguments constrain it to be of non-baryonic origin, and a vast amount of extensions to the Standard Model of particles has flourished in the literature, within which many new particles comply (with more or less fine tuning of theories) with the requirements that a dark matter (DM) candidate should fulfill.

A vastly popular class of models is that of the so called Weakly Interacting Massive Particles (WIMPs), a typical example of which are the Ligthest Supersymmetric Partner or Kaluza-Klein mode, that bear the remarkable properties to be stable (under conservation of $\mathrm{R}$ and K-parity, respectively) and self-annihilating. Intriguingly, the self-annihilation rate arising "naturally" for such candidates being subject only to weak interactions, would produce a relic density -if they are to produced thermally in the early Universe- compatible with that of DM in a $\Lambda$ CDM Universe. This has often being vividly referred to as "WIMP miracle", and the reader is addressed to recent reviews [1], for a more detailed discussion of the topic.

Once their abundance is "frozen", WIMPs can continue annihilating, with a rate which depends locally on the (square) density field. Annihilations into standard model particles in general does not bear remarkable effects, as the energy released by annihilations is not comparable with the energy involved in "standard" processes. However, some cases in which DM annihilation can leave a distinct mark can indeed be found and I am summarizing them in these proceedings:

(i) during primordial nucleosynthesis, when the temperature of the Universe is about $100 \mathrm{keV}$ $\gtrsim \mathrm{T} \gtrsim 0.0001 \mathrm{keV}$ the annihilation of WIMPs into baryons can modify the standard nucleosynthetic outcomes, thus leaving an identifiable mark on the initial chemical composition of the Universe;

(ii) the effects of DM annihilation into the thermal, recombined gas at $10002 \mathrm{z} \approx 150$ has the effect to modify its temperature and ionization status, thus contributing to the amount of free electrons in the Early Universe;

(iii) during the first star formation episode in the Universe, WIMP DM annihilation may affect both the proto-stellar phase and, once they form, the evolution of the first stars (by means of different mechanisms). This may in principle leave quite strong signatures on the first generation of stars (Population III).

The readers are addressed to the original literature for quantitative assesments and all concerning caveats, these Proceedings are intended only as a general review.

\section{Altering Primordial Nucleosynthesis}

Before the formation of gravitationally bound structures, the DM density field can be approximated by a smooth, diffuse one and the annihilation rate per unit volume, $A(\mathrm{z})$ reads:

$$
A(z)=\frac{1}{2} \rho_{c}^{2} \Omega_{D M}^{2}(1+z)^{6} \frac{\langle\sigma v\rangle(z)}{m_{\chi}^{2}}
$$


with $n_{D M}(z)$ being the relic DM abundance at a given redshift $z, m_{\chi}$ the mass of the dark matter particle, $\Omega_{D M}$ the cold dark matter fraction, $\rho_{c}$ the critical density of the Universe today, and $\langle\sigma v\rangle(\mathrm{z})$ is the effective self-annihilation rate which for the sake of generality here we assume to depend on the redshift $z$. The injection of high energy primaries into the plasma undergoing Primordial Nucleosynthesis (or Big Bang Nucleosynthesis-BBN) is to induce non-thermal processes that eventually affect the final nucleosynthetic outcome. The hadronic and electromagnetic shower injected in the plasma do in fact create spallation reactions and annihilation of antinucleons with the existing nuclei, and alter the neutron to proton ratio if injected early enough; quantitative assessment of this statement are reviewed in [3]. It is remarkable that high energy nuclei (with energies well above thermal ones, produced in the annihilating DM shower) can spallate onto ${ }^{4} \mathrm{He}$ producing ${ }^{2} \mathrm{H},{ }^{3} \mathrm{H}$ and ${ }^{3} \mathrm{He}$, with a final effect of an additional synthesis of ${ }^{6} \mathrm{Li}$. This has been thorouhly studied and clarified in [2], where also the necessity of the hadronic nature of the primary shower in order to produce additional ${ }^{6} \mathrm{Li}$ was recognized. It is intriguing that WIMP DM candidates (annihlating into hadronic standard model particles) with self annihilation cross sections $\langle\sigma v\rangle=10^{-25} \mathrm{~cm}^{3} / \mathrm{s}$ (just slightly above the benchmark value of the "vanilla" thermal WIMP scenario, $\langle\sigma v\rangle=3 \times 10^{-26} \mathrm{~cm}^{3} / \mathrm{s}$ ) and masses above $\mathrm{m}_{\chi} \sim 50 \mathrm{GeV}$ produce a primordial ${ }^{6} \mathrm{Li}$ abundance (see references in [3] for a review of observations, and a thorough discussion) compatible with recent observations, without altering the other primordial abundances.

\section{Reionizing the IGM}

After Recombination, the gas becomes more transparent to the high energy primary shower, and only part of the total energy $2 m_{\chi} \mathrm{c}^{2}$ produced in the annihilation will be injected into the thermal gas -to which I will refer in the following as Inter Galactic Medium (IGM): the remaining of the high energy shower produced in the annihilation will in fact not interact with the thermal gas and stream freely through the Universe. Under the so called "on-the-spot" approximation, consisting in the assumption that the particles failing to interact with the IGM on-the-spot (namely within a short fraction of the Hubble time at the moment they are produced), do not interact with the thermal gas anymore, the energy deposited at any given time will actually only be a fraction $f(\mathrm{z})$ of the one produced, bearing an energy injection rate per unit volume:

$$
\frac{d E}{d t}(z)=f(z) A(z)=f(z) \rho_{c}^{2} c^{2} \Omega_{D M}^{2}(1+z)^{6} \frac{\langle\sigma v\rangle(z)}{m_{\chi}},
$$

the fraction $f(z)$ depending on the nature of the high energy primary shower, and being directly computable for each DM candidate, being due only to well known high energy astrophysical processes (inverse Compton scattering, Pair Production on CMB photons etc., see [6].)

Once part of the initial energy due to the annihilations has been degraded down to the $\mathrm{keV}$ scale, the effects of such a low-energy, yet non thermal component are well known: in [4], the authors showed that the final effects are to provide (i) ionization, (ii) heating and (iii) Ly- $\alpha$ excitation of the thermal gas, the details and final ripartition of the three processes eventually depending only on the temperature and original ionization fraction of the affected gas, and recently [5] provided more accurate estimates in light of detailed MonteCarlo simulations. Many authors have modified the publicly available code RECFAST, which computes the properties of the evolving thermal gas 
in a cosmological context, by taking into account additional sources of ionization, see [7] for a non comprehensive list of the authors that have recently contributed to this field. By contributing to secondary ionization of the IGM, some WIMP candidates are able to produce a sizable fraction of the electron optical depth $\tau_{e}$ which is formally written as:

$$
\tau_{e}=-\int n_{e}(z) \sigma_{\mathrm{T}} d z
$$

$\mathrm{n}_{e}(z)$ being the fractional abundance of free electrons at redshift $z$ and $\sigma_{T}$ the Thomson scattering cross-section. The authors of [8] did in fact recently show that WIMPs with masses $m_{\chi}$ between the $\mathrm{GeV}$ and few $\mathrm{TeV}$, and self-annihilation cross sections compatible with the "vanilla" WIMP DM scenario, can even overproduce the amount of free electrons inferred through the CMB spectra observations, with the only contribution of the smooth, pre-structure formation component of the DM density field, the latter being negligible, [9]. . The actual value of mass and $\langle\sigma v\rangle$ depends on the nature of the shower produced during the annihilation, as leptons and handrons behave and hence couple differently with the IGM, as mentioned above. The non-detection in the WMAP5 data of the modifications implied by a high $\tau_{e}$ on the CMB spectra by very high self-annhilation rate has been used in order to place constraints in the WIMP $\langle\sigma v\rangle$ vs $\mathrm{m}_{\chi}$ plane; however, many models still compatible with a wealth of astrophysical are able to reproduce sizable amounts of the $\tau_{e}$. For instance, thermally decoupled WIMPs (i.e. $\langle\sigma v\rangle=3 \times 10^{-26} \mathrm{~cm}^{3} / \mathrm{s}$ ) and mass $\mathrm{m}_{\chi}=100 \mathrm{GeV}$ annihilating mainly into $\mathrm{e}^{+} \mathrm{e}^{-}$can reproduce a $\tau_{e}=0.002$, which is one third of the conservative estimate for the $\tau_{e}$ produced by unknown sources at redshift $\mathrm{z}>6$. A $\mathrm{m}_{\chi}=20 \mathrm{GeV}$ WIMP annihilating in the same channel and with the same $\langle\sigma v\rangle$ can reproduce the whole $\tau_{e}$ at $\mathrm{z}>6$ This is extremely interesting in terms of high redshift astrophysical sources. The Epoch of Reionization (EoR) is feebly being explored so far in terms of astrophysical sources, and many paramaters of the astrophysical populations are left free, often tuned in order not to overproduce ionization at high redshift. The presence of DM in the form of specific candidates may therefore reduce the room for astrophysics at high redshift, with a consequent need for rielaboration of the scenarios.

\section{Powering the first stars}

The first stars to form in a $\Lambda \mathrm{CDM}$ Universe are believed to be hosted within small mass halos at high redshift ( $\mathrm{z} \sim 10)$; the absence of metals and of big magnetic fields suggest that the cooling and consequente collapse of the gas cloud after the virialization is smooth, thus obtaining the double effect that the star forms in the very center of the halo, and that its mass will be in the tens to hundreds solar masses regime $\left(30-300 \mathrm{M}_{\odot}\right)$.

In [10] the authors first noticed that the collapse of the gas should drag DM beyond, and the build-up of a more DM dense region where the proto-star forms. The enhanced gas opacity to the high energy annihilation induced shower conspires, together with the enhanced annihilation rate, to a dramatic raise in a DM energy injection in the gas cloud, which was found to be at the level of the cooling term due to gas collisions, for several combinations of WIMP masses and self-annihilation cross sections. Studies have confirmed this picture, [11], finding at the same time that this have no dramatic effects on stellar formation, [12]; this very same mechanism of DM contraction can slow down the collapse of a hydrostatic object when it is already close to igniting nuclear reactions 
[13]. It is yet unclear if this mechanism can leave an imprint on the initial mass function (IMF) of the first stars by allowing dramatic accretion onto the cold hydrostatic object while it reaches he Zero Age Main Sequence, [13, 14], thus resulting in a primordial IMF peaked around very massive objects $\left(1000 \mathrm{M}_{\odot}\right)$.

Whichever final object will result from this mechanism, once it is very close to igniting nuclear reactions (close to the Zero Age Main Sequence), WIMPs originally external to the star, that cross it can be scattered off the nuclei constituting the star and be captured by losing energy in the scatter, cumulating in the very center of the star. At the very high DM densities primordial stars leave in, this mechanism of capture and cumulation (and subsequent annihilation) can provide an amount of energy comparable with the one needed to support the star, and usually provided for by nuclear reactions, [15]- for values of the spin-dependent elastic scattering cross-section at the current upper limits. The main effect of this would be the life prolongement of primordial stars [16], which could therefore survive for longer times than the $10^{6}-10^{7} \mathrm{yr}$ to which their mass would condemn them to. Whether this could lead to direct observables able to prove or disprove the effects of WIMP DM annihilations onto the first stars is under study, [17], and the forthcoming JWST seems the most obvious instrument with which address the question.

\subsection{Conclusions}

I have briefly summarized the main three ways in which Weakly Interacting Massive Particles, if they are to constitute the bulk of Dark Matter, can leave macroscopic imprints on quantities of concern of Cosmology with the products of their self-annihilations. The alteration of the outcome of Primordial Nucleosynthesis, and in particular of the ${ }^{6} \mathrm{Li}$ abundance is possible within some models (annihilating into a hadronic shower); some of this very same models (as well as many others annihilating mainly into leptons) should also induce enough secondary ionization into the thermal gas after Recombination, thus leaving an imprint in the CMB spectra. Most dramatic is the effect that WIMPs may have on the formation and the evolution of the first stars: models with a spin-dependent elastic scattering cross sections (between WIMPs and baryons) at the level of the current upper limits may induce extremely prolonged lifetimes of the first stars in the Universe, thus changing our view of the high redshift astrophysics.

\section{References}

[1]G. Jungman, M. Kamionkowski and K. Griest, Phys. Rept. 267, 195 (1996), [arXiv:hep-ph/9506380]; L. Bergstrom, Rep. Prog. Phys. 63, 793 (2000), [arXiv:hep-ph/0002126]; G. Bertone, D. Hooper and J. Silk, Phys. Rept. 405, 279 (2005), [arXiv:hep-ph/0404175].

[2]K. Jedamzik, Phys. Rev. D 70 (2004) 083510 [arXiv:astro-ph/0405583].

[3]F. Iocco, G. Mangano, G. Miele, O. Pisanti and P. D. Serpico, Phys. Rept. 472 (2009) 1 [arXiv:0809.0631 [astro-ph]].

[4]J. M. Shull and M. E. van Steenberg, Astroph. Journ. 298 (1985) 268.

[5]M. Valdes and A. Ferrara, Mon. Not. Roy. Astron. Soc. 387L, 8V (2008) arXiv:0803.0370 [astro-ph].

[6]T. R. Slatyer, N. Padmanabhan and D. P. Finkbeiner, Phys. Rev. D 80 (2009) 043526 [arXiv:0906.1197 [astro-ph.CO]]. 
[7]R. Bean, A. Melchiorri and J. Silk, Phys. Rev. D 68 (2003) 083501 [arXiv:astro-ph/0306357];

N. Padmanabhan and D. P. Finkbeiner, Phys. Rev. D 72 (2005) 023508 [arXiv:astro-ph/0503486];

L. Zhang, X. L. Chen, Y. A. Lei and Z. G. Si, Phys. Rev. D 74, 103519 (2006),

[arXiv:astro-ph/0603425]; A. Lewis, J. Weller, and R. Battye, Mon. Not. Roy. Astron. Soc. 373, 561 (2006) [arXiv:astro-ph/0606552]; M. Mapelli, A. Ferrara and E. Pierpaoli, Mon. Not. Roy. Astron. Soc. 369, 1719 (2006) [arXiv:astro-ph/0603237]; S. Galli, R. Bean, A. Melchiorri and J. Silk, Phys. Rev. D 78 (2008) 063532 [arXiv:0807.1420 [astro-ph]]; J. Kim and P. Naselsky, arXiv:0802.4005 [astro-ph];

S. Galli, F. Iocco, G. Bertone and A. Melchiorri, Phys. Rev. D 80, 023505 (2009) [arXiv:0905.0003 [astro-ph.CO]].

[8]G. Huetsi, A. Hektor and M. Raidal, arXiv:0906.4550 [astro-ph.CO]; M. Cirelli, F. Iocco and P. Panci, JCAP 0910 (2009) 009 [arXiv:0907.0719 [astro-ph.CO]].

[9]A. Natarajan and D. J. Schwarz, Phys. Rev. D 78, 103524 (2008); [arXiv:0805.3945 [astro-ph]]. A. Natarajan and D. J. Schwarz, Phys. Rev. D 80, 043529 (2009), [arXiv:0903.4485 [astro-ph.CO]].

[10]D. Spolyar, K. Freese and P. Gondolo, Phys. Rev. Lett. 100 (2008) 051101 [arXiv:0705.0521 [astro-ph]].

[11]A. Natarajan, J. C. Tan and B. W. O’Shea, Astrophys. J. 692 (2009) 574 [arXiv:0807.3769 [astro-ph]].

[12]E. Ripamonti, F. Iocco, A. Bressan, R. Schneider, A. Ferrara and P. Marigo, PoS IDM08 (2009) 75 [arXiv:0903.0346 [astro-ph.CO]]; E. Ripamonti, F. Iocco, A. Bressan, R. Schneider, A. Ferrara and P. Marigo, work in progress.

[13]F. Iocco, A. Bressan, E. Ripamonti, R. Schneider, A. Ferrara and P. Marigo, Mon. Not. Roy. Astron. Soc. 390 (2008) 1655 [arXiv:0805.4016 [astro-ph]].

[14]K. Freese, P. Bodenheimer, D. Spolyar and P. Gondolo, Astrophys. J. 685 (2008) L101 [arXiv:0806.0617 [astro-ph]]; K. Freese, D. Spolyar, P. Bodenheimer and P. Gondolo, New J. Phys. 11 (2009) 105014 [arXiv:0903.0101 [astro-ph.CO]].

[15]F. Iocco, Astrophys. J. 677 (2008) L1 [arXiv:0802.0941 [astro-ph]]; K. Freese, D. Spolyar and A. Aguirre, JCAP 0811 (2008) 014 [arXiv:0802.1724 [astro-ph]].

[16]S. C. Yoon, F. Iocco and S. Akiyama, Astrophys. J. 688 (2008) L1 [arXiv:0806.2662 [astro-ph]]; M. Taoso, G. Bertone, G. Meynet and S. Ekstrom, Phys. Rev. D 78 (2008) 123510 [arXiv:0806.2681 [astro-ph]].

[17]F. Iocco, Nucl. Phys. Proc. Suppl. 194 (2009) 82 [arXiv:0906.4106 [astro-ph.CO]]; E. Zackrisson et al., work in progress. 